\title{
On Regular Mildly Generalized (RMG) Open Sets in Topological Spaces
}

\author{
R. S. Wali ${ }^{1}$, Nirani Laxmi ${ }^{2}$ \\ ${ }^{I}$ Department of Mathematics, Bhandari and Rathi College, Guledagudd-587 203, Karnataka- India. \\ ${ }^{2}$ Department of Mathematics, Rani Channamma University, Belagavi-591 156, Karnataka-India.
}

\begin{abstract}
In this paper we introduce and study the new class of sets, namely Regular Mildly Generalized Open (briefly, RMG-open) sets, Regular Mildly Generalized neighborhoods (briefly, RMG-nhd), RMG-interior and $R M G$-closure in topological space and also some properties of new concept has been studied.

Keywords: RMG-closed sets, RMG-open sets, RMG-neighborhoods, RMG-interior, RMG-closure.
\end{abstract}

\section{Introduction}

Levine $[9,10]$ introduces a generalized open and semi-open sets in topological spaces. Regular open sets, pre-open sets, rg-open sets, Mildly-g-open sets have been introduced and studied by stone [18], A.S. Mashhur.et.al[5], N. Palaniappan et. al[14], J. K. Park et. al[16] respectively. In this paper the concept of Regular Mildly Generalized (briefly RMG) open set is introduced and their properties are investigated and Regular Mildly Generalized neighborhood (briefly RMG-nhd), RMG-interior and RMG- closure in a topological spaces.

Throughout this paper $\mathrm{X}$ and $\mathrm{Y}$ represent the topological spaces on which no separation axioms are assumed unless otherwise mentioned. For a subset $A$ of topological space $X, \operatorname{cl}(A)$ and $\operatorname{int}(A)$ denote the closure of $\mathrm{A}$ and interior of $\mathrm{A}$ respectively. $\mathrm{X}-\mathrm{A}$ or $\mathrm{A}^{\mathrm{c}}$ Denotes the complement of $\mathrm{A}$ in $\mathrm{X}$. Now, we recall the following definitions.

\section{Preliminaries}

Definition 2.1 A subset A of $X$ is called regular open (briefly r-open) [18] set if $A=\operatorname{int}(\mathrm{cl}(\mathrm{A})$ ) and regular closed (briefly $\mathrm{r}$-closed) [18] set if $\mathrm{A}=\operatorname{cl}(\operatorname{int}(\mathrm{A})$ )).

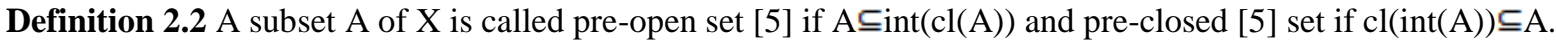

Definition 2.3 A subset $A$ of $X$ is called semi-open set [8] if $A \subseteq c l(i n t(A))$ and semi-closed [8] set if $\operatorname{int}(\mathrm{cl}(\mathrm{A})) \subseteq \mathrm{A}$.

Definition 2.4 A subset A of $X$ is called $\alpha$-open [11] if $A \subseteq \operatorname{int}(\operatorname{cl}(\operatorname{int}(A)))$ and $\alpha$-closed [11] if $\operatorname{cl}(\operatorname{int}(\operatorname{cl}(A))) \subseteq A$.

Definition 2.5 A subset $A$ of $X$ is called $\beta$-open [1] if $A \subseteq \operatorname{cl}(\operatorname{int}(\operatorname{cl}(A)))$ and $\beta$-closed [1] if $\operatorname{int}(\operatorname{cl}(\operatorname{int}(A))) \subseteq A$.

Definition 2.6 A subset $A$ of $X$ is called $\delta$-closed [19] if $A=\operatorname{cl}_{\delta}(A)$, where $\operatorname{cl}_{\delta}(A)=\{x \in X: \operatorname{int}(\operatorname{cl}(U)) \cap A \neq$ $\emptyset, \mathrm{U} \in \mathrm{A}\}$.

Definition 2.7 Let $X$ be a topological space. The finite union of regular open sets in $X$ is said to be $\pi$-open [4]. The compliment of a $\pi$-open set is said to be $\pi$-closed.

Definition 2.8 A subset of a topological space $(X, \tau)$ is called

1. Generalized closed (briefly g-closed) [9] if $\operatorname{cl}(\mathrm{A}) \subseteq \mathrm{U}$ whenever $\mathrm{A} \subseteq \mathrm{U}$ and $\mathrm{U}$ is open in $\mathrm{X}$.

2. Generalized $\alpha$-closed (briefly g $\alpha$-closed) [6] if $\alpha$-cl(A) $\subseteq U$ whenever $A \subseteq U$ and $U$ is $\alpha$-open in $X$.

3. Weakly generalized closed (briefly wg-closed) [10] if $\operatorname{cl}(\operatorname{int}(\mathrm{A})) \subseteq \mathrm{U}$ whenever $\mathrm{A} \subseteq \mathrm{U}$ and $\mathrm{U}$ is open in $\mathrm{X}$.

4. Strongly generalized closed (briefly $\mathrm{g}^{*}$-closed) [15] if $\mathrm{cl}(\mathrm{A}) \subseteq \mathrm{U}$ whenever $\mathrm{A} \subseteq \mathrm{U}$ and $\mathrm{U}$ is g-open in $\mathrm{X}$.

5. Weakly closed (briefly w-closed) [17] if cl(A) $\subseteq U$ whenever $A \subseteq U$ and $\mathrm{U}$ is semi-open in $\mathrm{X}$.

6. Mildly generalized closed (briefly mildly g-closed) [16] if $\operatorname{cl}(\operatorname{int}(\mathrm{A})) \subseteq \mathrm{U}$ whenever $\mathrm{A} \subseteq \mathrm{U}$ and $\mathrm{U}$ is g-open in $\mathrm{X}$.

7. Regular weakly generalized closed (briefly rwg-closed) [10] if $\operatorname{cl}(\operatorname{int}(\mathrm{A})) \subseteq \mathrm{U}$ whenever $\mathrm{A} \subseteq \mathrm{U}$ and $\mathrm{U}$ is regular open in X.

8. Weakly $\pi$-generalized closed (briefly w $\pi \mathrm{g}$-closed)[13] if $\operatorname{cl}(\operatorname{int}(\mathrm{A})) \subseteq \mathrm{U}$ whenever $\mathrm{A} \subseteq \mathrm{U}$ and $\mathrm{U}$ is $\pi$-open in $\mathrm{X}$.

9. Regular weakly closed (briefly rw-closed)[2] if $\mathrm{cl}(\mathrm{A}) \subseteq \mathrm{U}$ whenever $\mathrm{A} \subseteq \mathrm{U}$ and $\mathrm{U}$ is regular semiopen in $\mathrm{X}$.

10. Generalized pre closed (briefly gp-closed)[7] if pcl(A) $\subseteq \mathrm{U}$ whenever $\mathrm{A} \subseteq \mathrm{U}$ and $\mathrm{U}$ is open in $\mathrm{X}$.

11. A subset $A$ of a space $(X, \tau)$ is called regular generalized closed (briefly rg-closed) [14] if $\operatorname{cl}(\mathrm{A}) \subseteq \mathrm{U}$ whenever $\mathrm{A} \subseteq \mathrm{U}$ and $\mathrm{U}$ is regular open set in $\mathrm{X}$. 
12. $\pi$-generalized closed (briefly $\pi g$-closed)[3] if $\operatorname{cl}(\mathrm{A}) \subseteq \mathrm{U}$ whenever $\mathrm{A} \subseteq \mathrm{U}$ and $\mathrm{U}$ is open in $\mathrm{X}$.

The complements of the above mentioned closed sets are their respective open sets.

The semi-pre-closure (resp. semi-closure, resp. pre-closure, resp. $\alpha$-closure) of a subset A of X is the intersection of all semi-pre- closed (resp. semi- closed, resp. pre- closed, resp. $\alpha$-closed) sets containing A and is denoted by $\operatorname{spcl}(\mathrm{A})($ resp. $\operatorname{scl}(\mathrm{A})$, resp. $\operatorname{pcl}(\mathrm{A})$, resp. $\operatorname{cl}(\mathrm{A})$ ).

Definition 2.3 Regular Mildly Generalized closed (briefly RMG-closed)[20] if $\operatorname{cl}(\operatorname{int}(\mathrm{A})) \subseteq \mathrm{U}$ whenever A $\subseteq \mathrm{U}$ and $\mathrm{U}$ is rg-open in $\mathrm{X}$. We denote the family of all RMG-closed sets, RMG-open sets of X by RMGC(X), $\mathrm{RMGO}(\mathrm{X})$ respectively.

\section{Regular Mildly Generalized Open (briefly RMG-open) Sets}

In this section, we introduce and studied RMG-open sets in topological space and obtain some of their basic properties. Also we introduce RMG-neighborhood (briefly RMG-nhd) in topological spaces by using the notation of RMG-open sets.

Definition 3.1: A subset A of $X$ is called Regular Mildly Generalized open (briefly, RMG-open) set in X. If $\mathrm{X}-\mathrm{A}$ is RMG-closed set in X. The family of all RMG- open sets is denoted by RMGO(X).

Theorem 3.2: Every pre-open set is RMG-open set in $X$.

Proof: Let A be a pre-open set in X. Then by X - A is pre-closed. By Theorem 3.2[20] every pre-closed set is RMG-closed, $\mathrm{X}-\mathrm{A}$ is RMG-closed set in X. Therefore A is RMG-open set in X.

The converse of the above theorem need not be true as seen from the following example.

Example3.3: Let $X=\{a, b, c, d\}$ with topology $\tau=\{X, \emptyset,\{a\},\{b\},\{a, b\},\{b, c\},\{a, b, c\}\}$.Then $A=\{c\}$ is RMGopen set but not pre-open set in $\mathrm{X}$.

Theorem3.4: Every RMG-open set is Mildly-g-open set in X.

Proof: Let A be a RMG-open set in X. Then X - A is RMG -closed. By Theorem 3.4[20] Every RMG-closed set is Mildly-g-closed, X - A is RMG-closed. Therefore A is Mildly-g-open set in X.

The converse of above Theorem need not be true as seen from the following examples.

Examples3.5: Let $X=\{a, b, c, d\}$ with topology $\tau=\{X, \varnothing,\{a\},\{b\},\{a, b\},\{a, b, c\}\}$.Then $A=\{a c\}$ and $B=\{b, c\}$ are mildly-g-open set but not RMG-open set in X.

Corollary 3.6: 1. Every g $\alpha$-open set is RMG-open set in X.

2. Every w-open set is RMG-open set in $X$.

3. Every open set is RMG-open set in X.

4. Every 8 -open set is RMG-open set in X.

5. Every $\pi$-open set is RMG-open set in $X$.

6. Every regular open set is RMG-open set in $\mathrm{X}$.

Proof:

1. Let $\mathrm{A}$ be a $\mathrm{g} \alpha$-open set in $\mathrm{X}$. Then $\mathrm{X}-\mathrm{A}$ is $\mathrm{g} \alpha$-closed set. By Theorem 3.6.1[20] every g $\alpha$-closed set is RMG- closed, $\mathrm{X}-\mathrm{A}$ is RMG-closed .Therefore A is RMG-open set in $\mathrm{X}$.

2. Let $\mathrm{A}$ be a w-open set in $\mathrm{X}$. Then $\mathrm{X}-\mathrm{A}$ is $\mathrm{w}$-closed. By Theorem 3.6.2[20] every w-closed set is RMGclosed, $\mathrm{X}-\mathrm{A}$ is RMG-closed .Therefore A is RMG-open set in $\mathrm{X}$.

3. Let A be a open set in X. Then X - A is closed. By Theorem 3.6.3[20] every closed set is RMG-closed, X - A is RMG-closed .Therefore $\mathrm{A}$ is RMG-open set in $\mathrm{X}$.

4. Let A be a $\delta$-open set in X. Then X - A is $\delta$-closed. By Theorem 3.6.4[20] every $\delta$ - closed set is RMG-closed, $\mathrm{X}-\mathrm{A}$ is RMG-closed .Therefore $\mathrm{A}$ is RMG-open set in $\mathrm{X}$.

5. Let $A$ be a $\pi$-open set in $X$. Then $X-A$ is $\pi$-closed. By Theorem 3.6.5[20] every $\pi$ - closed set is RMGclosed, $\mathrm{X}-\mathrm{A}$ is RMG-closed .Therefore $\mathrm{A}$ is RMG-open set in $\mathrm{X}$.

6. Let $\mathrm{A}$ be a regular open set in $\mathrm{X}$. Then $\mathrm{X}-\mathrm{A}$ is regular closed. By Theorem 3.6.6[20] every regular closed set is RMG-closed, $\mathrm{X}-\mathrm{A}$ is RMG-closed .Therefore A is RMG-open set in $\mathrm{X}$.

The converse of Corollary 3.6 is not true as shown in below examples.

Example 3.7: Let $X=\{a, b, c, d\}$ with topology $\tau=\{X, \emptyset,\{a\},\{b\},\{a, b\},\{b, c\},\{a, b, c\}\}$.

1. Let $A=\{c\}$ is RMG-open but not g $\alpha$-open set in $X$.

2. Let $A=\{c\}$ is RMG-open but not w-open set in $X$.

3. Let $A=\{c\}$ is RMG-open but not open set in $X$.

4. Let $A=\{c\}$ is RMG-open but not 8 -open set in $X$.

5. Let $A=\{c\}$ is RMG-open but not $\pi$-open set in $X$.

6. Let $A=\{c\}$ is RMG-open but not regular open set in $X$.

Corollary 3.8:

1. Every RMG-open set is wg-open set in X. 


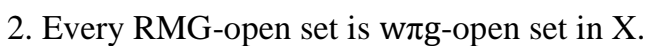

3. Every RMG-open set is rwg-open set in X.

Proof: 1. Let A be a RMG-open set in X. Then X - A is RMG-closed. By Theorem 3.8.1[20] every RMGclosed set is wg-closed, $\mathrm{X}-\mathrm{A}$ is RMG-closed. Therefore $\mathrm{A}$ is wg-open set in $\mathrm{X}$.

2. Let A be a RMG-open set in X. Then $X-A$ is RMG-closed. By Theorem 3.8.2[20] every RMG-closed set is witg-closed, $\mathrm{X}-\mathrm{A}$ is RMG-closed. Therefore A is wig-open set in X.

3. Let A be a RMG-open set in X. Then X - A is RMG-closed. By Theorem 3.8.3[20] every RMG- closed set is rwg-closed, $\mathrm{X}-\mathrm{A}$ is rwg-closed. Therefore $\mathrm{A}$ is rwg-open set in $\mathrm{X}$.

The converse of corollary 3.8 is not true as shown in below examples.

Example3.9: Let $X=\{a, b, c, d\}$ with topology $\tau=\{X, \emptyset,\{a\},\{b\},\{a, b\},\{a, b, c\}\}$.

1. Let $A=\{c\}$ is wg-open but not RMG-open set in $X$.

2. Let $A=\{b, d\}$ is w $\pi$ g-open but not RMG-open set in $X$.

3. Let $A=\{d\}$ is rwg-open but not RMG-open set in $X$.

Remark 3.10: The concept of semi-open, semi-pre-open, g-open, $g^{*}$-open, rg-open, rw-open, $\pi g$-open sets are independent with the concept of RMG-open sets as shown in the following example.

Example 3.11: Let $X=\{a, b, c, d\}$ with topology $\tau=\{X, \varnothing,\{a\},\{b\},\{a, b\},\{b, c\},\{a, b, c\}\}$. Then the set $\{a, b$, $\mathrm{d}\}$ is RMG-open set. However it can be verified that it is not $\mathrm{g}$-open, not $\mathrm{g}^{*}$-open, not $\pi \mathrm{g}$-open, not rg-open, not rw-open,, Also $\{b, d\}$ is both semi-open and semi pre open but not RMG-open .

Remark 3.12From the above discussions and known results we have the following implications in the following diagram, by

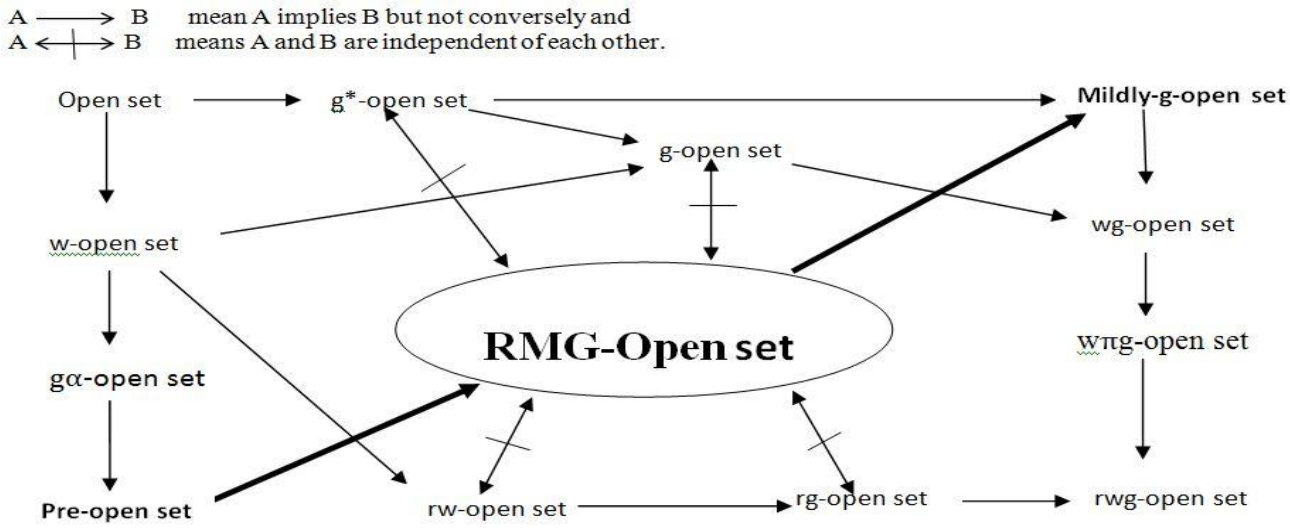

Remark s3.13: The intersection of two RMG-open sets in $X$ is need not be a RMG- open set in X.

Examples 3.14: Let $X=\{a, b, c, d\}$ with topology $\tau=\{X, \varnothing,\{a\},\{b, c\},\{a, b, c\}\}$. Now $A=\{a, b, d\}$ and $B=\{a$, $c, d\}$ are $R M G$-open sets in $X$, then $A \cap B=\{a, b, d\} \cap\{a, c, d\}=\{a, d\}$ which is not RMG- open set in $X$

Remark 3.15: The union of two RMG-open subsets of $X$ is need not be RMG-open set in $X$.

Example 3.16: Let $X=\{a, b, c, d\}$ with topology $\tau=\{X, \emptyset,\{a\},\{b\},\{a, b\},\{b, c\},\{a, b, c\}\}$. Now $A=\{a\}$ and $\mathrm{B}=\{\mathrm{c}\}$ are RMG-open sets in $\mathrm{X}$, then $\mathrm{A} \cup \mathrm{B}=\{\mathrm{a}\} \cup\{\mathrm{c}\}=\{\mathrm{a}, \mathrm{c}\}$ which is not RMG-open set in $\mathrm{X}$.

Remark 3.17: Complement of a RMG-open set need not be RMG-open set in X.

Example 3.18: Let $X=\{a, b, c, d\}$ with topology $\tau=\{X, \emptyset,\{a\},\{b\},\{a, b\},\{b, c\},\{a, b, c\}\}$. Then $A=\{a, b\}$ is RMG-open set but $X-\{a, b\}=\{c, d\}$ is not RMG-open set in $X$.

Theorem 3.19: A subset $A$ of a topological space $X$ is RMG-open iff $F \subset i n t(c l(A))$ whenever $F \subset A$ and $F$ is rgclosed set in X.

Proof: Assume A is RMG-open then $\mathrm{X}-\mathrm{A}$ is RMG-closed. Let $\mathrm{F}$ be a rg-closed set in $\mathrm{X}$ contained in A. Then $X-F$ is a $\operatorname{rg}$-open set in $X$ containing $X-A$. Since $X-A$ is RMG-closed, $\operatorname{cl}(\operatorname{int}(X-A)) \subset X-F$ this implies $X-\operatorname{int}(\operatorname{cl}(A)) \subset X-F$. Consequently F $\subset \operatorname{int}(\mathrm{cl}(\mathrm{A}))$.

Conversely, let $\mathrm{F} \subset \operatorname{int}(\mathrm{cl}(\mathrm{A}))$ whenever $\mathrm{F} \subset \mathrm{A}$ and $\mathrm{F}$ is rg-closed in $\mathrm{X}$. Let $\mathrm{G}$ be $\operatorname{rg}$-open set containing $\mathrm{X}-\mathrm{A}$ then $\mathrm{X}-\mathrm{G} \subset \operatorname{int}(\mathrm{cl}(\mathrm{A}))$. Hence $\operatorname{cl}(\operatorname{int}(\mathrm{X}-\mathrm{A})) \subset \mathrm{G}$. This prove that $\mathrm{X}-\mathrm{A}$ is RMG-closed and hence A is RMG-open set in $\mathrm{X}$.

Theorem 3.20: If $A \subseteq X$ is RMG-closed set in $X$, then $\operatorname{cl}(\operatorname{int}(A))-A$ is RMG-open set in $X$.

Proof: Let $\mathrm{A} \subseteq \mathrm{X}$ is $\mathrm{RMG}$-closed and let $\mathrm{F}$ be a rg-closed set such that $\mathrm{F} \subseteq \mathrm{cl}(\operatorname{int}(\mathrm{A}))-\mathrm{A}$. Then by Theorem $3.21[20], \mathrm{F}=\emptyset$, that implies $\mathrm{F} \subseteq \operatorname{int}(\mathrm{cl}(\mathrm{cl}(\operatorname{int}(\mathrm{X}-\mathrm{A}))))-\mathrm{A}$. This proves that $\mathrm{cl}(\operatorname{int}(\mathrm{A}))-\mathrm{A}$ is RMG-open.

Theorem 3.21: Every singleton point set in a space $\mathrm{X}$ is either RMG-open or rg-closed. 
Proof: Let $X$ be a topological space. Let $\mathrm{x} \in \mathrm{X}$. To prove $\{\mathrm{x}\}$ is either RMG-open or rg-closed. That is to prove $\mathrm{X}-\{\mathrm{x}\}$ is either RMG-closed or rg-open. Which follows from Theorem 3.23 of [20].

Theorem 3.22: If $\operatorname{int}(\mathrm{cl}(\mathrm{A})) \subseteq \mathrm{B} \subseteq \mathrm{A}$ and $\mathrm{A}$ is RMG-open, then $\mathrm{B}$ is RMG-open.

Proof: Let $\mathrm{A}$ be RMG-open and $\operatorname{int}(\mathrm{cl}(\mathrm{A})) \subseteq \mathrm{B} \subseteq \mathrm{A}$. Then $\mathrm{X}-\mathrm{A} \subseteq \mathrm{X}-\mathrm{B} \subseteq \mathrm{X}-\operatorname{int}(\mathrm{cl}(\mathrm{A}))$ that implies $\mathrm{X}-\mathrm{A}$ $\subseteq \mathrm{X}-\mathrm{B} \subseteq \mathrm{cl}(\operatorname{int}(\mathrm{X}-\mathrm{A}))$. Since $\mathrm{X}-\mathrm{A}$ is RMG-closed, by Theorem 3.24 of [20] $\mathrm{X}-\mathrm{B}$ is RMG-closed. This proves that $\mathrm{B}$ is RMG-open.

The converse of above Theorem 3.24 need not be true in general.

Example 3.23: Let $X=\{a, b, c, d\}$ with topology $\tau=\{X, \varnothing,\{a\},\{b\},\{a, b\},\{a, b, c\}\}$. Now $A=\{a, b, c\}$ and $B=\{b, c\}$. Now $A$ and $B$ both are RMG-open sets. But int $(\mathrm{cl}(A)) \nsubseteq B \subseteq A$

Theorem 3.24: Let $A \subset Y \subset X$ and $A$ is RMG-open set in $X$. Then $A$ is RMG-open in $Y$ provided $Y$ is open set in X.

Proof: Let A be RMG-open in $\mathrm{X}$ and $\mathrm{Y}$ be a open sets in $\mathrm{X}$. Let $\mathrm{U}$ be any rg-open in $\mathrm{Y}$ such that $\mathrm{A} \subset \mathrm{U}$. Then

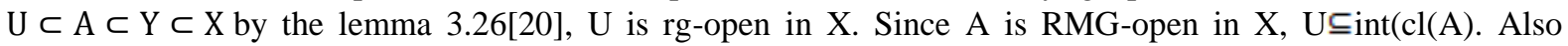
$\operatorname{int}(\mathrm{cl}(\mathrm{A})) \subset \operatorname{int}_{\mathrm{y}}\left(\mathrm{cl}_{\mathrm{y}}(\mathrm{A})\right)$. Hence $\mathrm{A}$ is an RMG-open set in $\mathrm{Y}$.

Theorem 3.25: If a subset $A$ is RMG-open in $X$ and if $G$ is $r$-open in $X$ with $\operatorname{int}(\operatorname{cl}(A) \cup(X-A) \subseteq G$ then $\mathrm{G}=\mathrm{X}$.

Proof: Suppose that $G$ is an $\operatorname{rg}$-open and $\operatorname{int}(\operatorname{cl}(A)) \cup(X-A) \subseteq G$. Now $(X-A) \subseteq(X-\operatorname{int}(\operatorname{cl}(A))) \cap X-$ $(X-A)$ implies that $(X-G) \subseteq \operatorname{cl}(\operatorname{int}(X-A)) \cap A$. Suppose $A$ is RMG-open. Since $X-G$ is rg-closed and $\mathrm{X}-\mathrm{A}$ is RMG-closed, then by Theorem 3.21 of [20], $\mathrm{X}-\mathrm{G}=\varnothing$ and hence $\mathrm{G}=\mathrm{X}$.

The converse of the above Theorem need not be true in general as shown in example 3.24.

Example3.26: Let $X=\{a, b, c, d\} \quad$ with topology $\tau=\{X, \varnothing,\{a\},\{b, c\},\{a, b, c\}\} \quad$. Then $\operatorname{RMGO}(X)=\{X, \varnothing,\{a\},\{b\},\{c\},\{a, b\},\{a, c\},\{b, c\},\{a, b, c\},\{a, b, d\},\{a, c, d\}\} \quad$ and $\operatorname{RGO}(X)=\{X, \varnothing,\{a\},\{b\},\{c\},\{d\},\{a, b\},\{a, c\},\{b, c\},\{b, d\},\{c, d\},\{a, b, c\}\}$. Let $A=\{b, d\}$ is not an RMG-open set in $X$. However $\operatorname{int}(\operatorname{cl}(A)) \cup(X-A)=\{b, c\} \cup\{a, c\}=\{a, b, c\}$. So for some rg-open set $G$, such that $\operatorname{int}(\mathrm{cl}(\mathrm{A})) \cup(\mathrm{X}-\mathrm{A})=\{\mathrm{a}, \mathrm{b}, \mathrm{c}\} \subset \mathrm{G}$ gives $\mathrm{G}=\mathrm{X}$ but $\mathrm{A}$ is not RMG-open set in $\mathrm{X}$.

Theorem3.27: Let $\mathrm{X}$ be a topological space and $\mathrm{A}, \mathrm{B} \subseteq \mathrm{X}$. If $\mathrm{B}$ is RMG-open and $\operatorname{int}(\operatorname{cl}(\mathrm{B})) \subseteq \mathrm{A}$, then $\mathrm{A} \cap \mathrm{B}$ is RMG-open in X.

Proof: Since B is RMG-open and $\operatorname{int}(\mathrm{cl}(\mathrm{B})) \subseteq \mathrm{A}$, then $\operatorname{int}(\mathrm{cl}(\mathrm{B})) \subseteq \mathrm{A} \cap \mathrm{B} \subseteq \mathrm{B}$, then by Theorem 3.24, $\mathrm{A} \cap \mathrm{B}$ is RMG-open set in $\mathrm{X}$.

\section{Regular Mildly Generalized Neighborhoods (briefly RMG-nhd)}

Definition 4.1 Let $(X, \tau)$ be a topological space and let $x \in X$. A subset $N$ is said to be RMG- neighborhood (briefly, RMG-nhd) of $x$, if and only if there exists an RMG-open set $G$ such that $x \in G \subset N$.

Definition 4.2(i) $A$ subset $N$ of $X$ is a RMG-nhd of $A \subseteq X$ in topological space $(X, \tau)$, if there exists an RMGopen set $\mathrm{G}$ such that $\mathrm{A} \subset \mathrm{G} \subset \mathrm{N}$.

(ii) The collection of all RMG-nhd of $\mathrm{x} \in \mathrm{X}$ is called RMG-nhd system at $\mathrm{x} \in \mathrm{X}$ and shall be denoted by RMG$\mathrm{N}(\mathrm{x})$.

Theorem 4.3: Every neighborhood $N$ of $x \in X$ is a RMG-nhd of $x$.

Proof: Let $\mathrm{N}$ be neighborhood of point $\mathrm{x} \in \mathrm{X}$. To prove that $\mathrm{N}$ is a RMG-nhd of $\mathrm{x}$. By definition of neighborhood, there exists an open set $G$ such that $x \in G \subset N$. As every open set is RMG-open, $G$ is an RMGopen set in X. Then there exists a RMG-open set $G$ such that $x \in G \subset N$. Hence $N$ is RMG-nhd of $x$.

Remark4.4: In general, a RMG-nhd $N$ of $x$ in $X$ need not be nhd of $x$ in $X$, as shown from example 4.5.

Example4.5: Let $X=\{a, b, c, d\}$ with topology $\tau=\{X, \varnothing,\{a\},\{b\},\{a, b\},\{b, c\},\{a, b, c\}\}$. Then $\operatorname{RMGO}(X)=$ $\{X, \emptyset,\{a\},\{b\},\{c\},\{a, b\},\{b, c\},\{a, b, c\},\{a, b, d\}\}$. The set $\{a, c\}$ is RMG-nhd of the point $c$, since the RMG-open set $\{c\}$ is such that $c \in\{c\} \subset\{a, c\}$. However, the set $\{a, c\}$ is not a neighbourhood of the point $c$, since no open set $G$ exists such that $c \in G \subset\{a, c\}$.

Theorem4.6: If a subset $\mathrm{N}$ of a space $\mathrm{X}$ is RMG-open, then $\mathrm{N}$ is a RMG-nhd of each of its points.

Proof: Suppose $\mathrm{N}$ is RMG-open. Let $\mathrm{x} \in \mathrm{N}$ we claim that $\mathrm{N}$ is a RMG-nhd of $\mathrm{x}$. For $\mathrm{N}$ is a RMG-open set such that $\mathrm{x} \in \mathrm{N} \subset \mathrm{N}$. Since $\mathrm{x}$ is an arbitrary point of $\mathrm{N}$, it follows that $\mathrm{N}$ is a RMG-nhd of each of its points.

Remark4.7: The converse of the above theorem is not true in general as seen from the following example 4.8.

Example4.8: Let $X=\{a, b, c, d\}$ with topology $\tau=\{X, \emptyset,\{a\},\{b\},\{a, b\},\{b, c\},\{a, b, c\}\}$. Then RMGO $(X)=$ $\{X, \emptyset,\{a\},\{b\},\{c\},\{a, b\},\{b, c\},\{a, b, c\},\{a, b, d\}\}$. The set $\{a, c\}$ is a RMG-nhd of the point a, since the RMGopen set $\{a\}$ is such that $a \in\{a\} \subset\{a, c\}$. Also the set $\{a, c\}$ is a RMG-nhd of the point $c$, since the RMG-open set $\{c\}$ is such that $c \in\{c\} \subset\{a, c\}$ i.e. $\{a, c\}$ is a RMG-nhd of each of its points. However the set $\{a, c\}$ is not a RMG-open set in $\mathrm{X}$. 
Theorem4.9: Let $X$ be a topological space. If $F$ is a RMG-closed subset of $X$ and $x \in(X-F)$, then there exists a RMG-nhd $\mathrm{N}$ of $\mathrm{x}$ such that $\mathrm{N} \cap \mathrm{F}=\emptyset$.

Proof: Let $F$ be RMG-closed subset of $X$ and $x \in(X-F)$. Then $(X-F)$ is an RMG-open set of $X$. By Theorem 4.6, $(X-F)$ contains a RMG-nhd of each of its points. Hence there exists a RMG-nhd $N$ of $x$ such that $N \subset X-$ F. That is $\mathrm{N} \cap \mathrm{F}=\emptyset$.

Theorem 4.10: Let $X$ be a topological space and for each $x \in X$, let RMG-N(x) be the collection of all RMGnhds of $x$. Then we have the following results.

i) $\forall x \in X, R M G-N(x) \neq \varnothing$.

ii) $N \in R M G-N(x) \Rightarrow x \in N$.

iii) $\mathrm{N} \in \mathrm{RMG}-\mathrm{N}(\mathrm{x})$ and $\mathrm{N} \subset \mathrm{M} \Rightarrow \mathrm{M} \in \mathrm{RMG}-\mathrm{N}(\mathrm{x})$.

iv) $N \in R M G-N(x) \Rightarrow \exists M \in R M G-N(x)$ such that $M \subset N$ and $M \in R M G-N(y)$ for every $y \in M$.

Proof: i) Since $X$ is an RMG-open set, it is a RMG-nhd of every $x \in X$. Hence $\exists$ at least one RMG-nhd(X) for each $\mathrm{x} \in \mathrm{X}$. Hence $\mathrm{RMG}-\mathrm{N}(\mathrm{x}) \neq \emptyset$ for every $\mathrm{x} \in \mathrm{X}$.

ii) If $N \in R M G-N(x)$, then $N$ is a RMG-nhd of $x$. So by definition of RMG-nhd $x \in N$.

iii) Let $N \in R M G-N(x)$ and $N \subset M$, then there is an RMG-open set $G$ such that $x \in G \subset N$. Since $N \subset M$, $x \in G \subset \quad M$ and so $M$ is a RMG-nhd of $x$. Hence $M \in R M G-N(x)$.

iv) If $N \in R M G-N(x)$, then there exists an RMG-open set $M$ such that $x \in M \subset N$. Since $M$ is an RMG-open set, it is a RMG-nhd of each of its points. Therefore $M \in R M G-N(y)$ for all $y \in M$.

\section{Regular Mildly Generalized Interior (RMG-Interior) Operator.}

In this section, the notation of RMG-interior is defined and some of its basic properties are studied.

Definition 5.1 (i): Let $A$ be a subset of $(X, \tau)$. A point $x \in A$ is said to be RMG-interior point of $A$ if and only if $\mathrm{A}$ is RMG-neighbourhood of $\mathrm{x}$. The set of all RMG-interior points of $\mathrm{A}$ is called the RMG-interior of $\mathrm{A}$ and is denoted by RMG-int(A).

Definition (ii): Let $(X, \tau)$ be a topological space and $A \subset X$. Then RMG-int (A) is the union of all RMG - open sets contained in A.

Theorem 5.2: Let $A$ is a subset of $(X, \tau)$, then $R M G-i n t(A)=U\{G: G$ is $R M G-o p e n, G \subset A\}$.

Proof: Let $A$ be a subset of $(X, \tau)$. X ERMG-int(A)

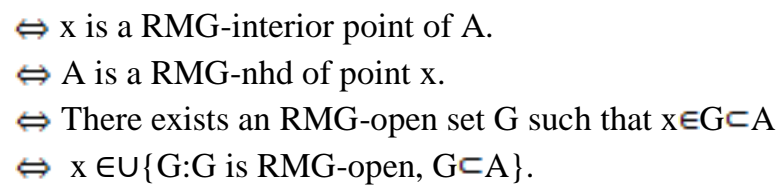

Hence $R M G-i n t(A)=U\{G: G$ is RMG-open $G \subset A\}$.

Theorem 5.3: Let $A$ and $B$ are subsets of $(X, \tau)$. Then

i) $\mathrm{RMG}$-int $(\varnothing)=\varnothing$ and $\mathrm{RMG}-\operatorname{int}(\mathrm{X})=\mathrm{X}$.

ii) $\mathrm{RMG}-\mathrm{int}(\mathrm{A}) \subset \mathrm{A}$.

ii) If $B$ is any $R M G-o p e n$ sets contained in $A$, then $B \subset R M G-i n t(A)$.

iv) If $A \subset B$, then $R M G$-int(A) $\subset R M G$-int(B).

v) RMG-int (RMG-int $(\mathrm{A}))=\mathrm{RMG}-\mathrm{int}(\mathrm{A})$.

Proof: i) Obvious

ii) Let $x \in R M G-i n t(A) \Rightarrow x$ is a RMG-interior point of $A$

$$
\begin{aligned}
& \Rightarrow \mathrm{A} \text { is a RMG-nhd of } \mathrm{x} \\
& \Rightarrow \mathrm{x} \in \mathrm{A}
\end{aligned}
$$

Thus $\mathrm{x} \in \mathrm{RMG}$-int(A) $\Rightarrow \mathrm{x} \in \mathrm{A}$. Hence RMG-int(A) $\subset \mathrm{A}$.

iii) Let $B$ be a any $R M G-o p e n$ set such that $B \subset A$. Let $x \in B$. Then since $B$ is an $R M G$ open set contained in $A$. $x$ is an $R M G$-interior point of $A$. That is $x \in R M G$-int(A). Hence $B \subset R M G-i n t(A)$.

iv) Let $A$ and $B$ are subsets of $X$ such that $A \subset B$. Let $x \in R M G-i n t(A)$. Then $x$ is an RMG-interior point of $A$ and so $A$ is a $R M G-n h d$ of $x$. since $A \subset B, B$ is also a RMG-nhd of $x$. This implies that $x \in R M G$-int(B). Thus we have show that $x \in R M G-i n t(A) \Rightarrow x \in R M G-i n t(B)$. Hence $R M G-i n t(A) \subset R M G-i n t(B)$.

v) Since RMG-int(A) is a RMG-open set in X, it follows that RMG-int(RMG-int(A))=RMG-int(A).

Theorem 5.4: If a subset $A$ of space $X$ is $R M G-o p e n$, then $R M G-i n t(A)=A$.

Proof: Let A be a RMG-open subset of $X$ and we know that RMG-int(A) $\subset A$. Since A is RMG-open set contained in A. From the Theorem 5.3(iii), $A \subset R M G-i n t(A)$ and hence we get RMG-int(A)=A.

The converse of the above theorem need not be true as seen in the following example. 
Example 5.5: Let $X=\{a, b, c, d\}$ with topology $\tau=\{X, \emptyset,\{a\},\{b\},\{a, b\},\{b, c\},\{a, b, c\}\}$. Then RMGO(X)= $\{X, \varnothing,\{a\},\{b\},\{c\},\{a, b\},\{b, c\},\{a, b, c\},\{a, b, d\}\}$. Note that RMG-int $(\{a, c\})=\{a\} \cup\{c\} \cup \emptyset=\{a, c\} . B u t\{a, c\}$ is not a RMG-open set in $\mathrm{X}$.

Theorem 5.6: If $A$ and $B$ are sub sets of $X$, then RMG-int(A)U RMG-int(B)ᄃ RMG-int(A UB).

Proof: Since $A \subset A \cup B$ and $B \subset A \cup B$. Using the Theorem 5.3(iv), RMG-int(A) $\subset$ RMG-int(AU B) and RMG$\operatorname{int}(B) \subset$ RMG-int(AUB)This implies RMG-int(A)U RMG-int(B) $\subset$ RMG-int(A UB).

Theorem5.7: If $A$ and $B$ are subsets of a space $X$, then RMG-int(AnB) $\subset$ RMG-int(A) $\cap R M G-i n t(B)$.

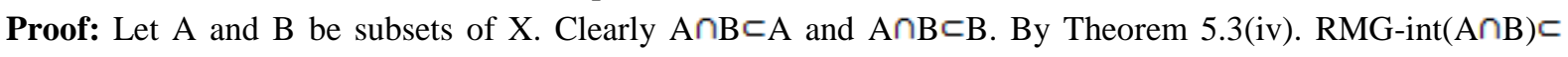

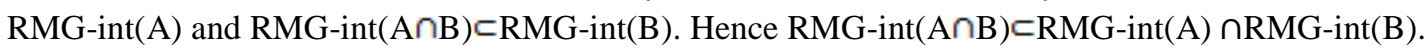

Theorem 5.8: If $A$ is a subset of $X$, then $\operatorname{int}(A) \subset R M G-i n t(A)$.

Proof: Let A be a subset of a space $X$.

$\mathrm{x} \in \operatorname{int}(\mathrm{A}) \Rightarrow \mathrm{x} \in \mathrm{U}\{\mathrm{G}: \mathrm{G}$ open, $\mathrm{G} \subset \mathrm{A}\}$.

$\Rightarrow$ There exists an open set $\mathrm{G}$ such that $\mathrm{x} \in \mathrm{G} \subset \mathrm{A}$

$\Rightarrow$ There exists an RMG-open set $\mathrm{G}$ such that $\mathrm{x} \in \mathrm{G} \subset \mathrm{A}$, as every open set is an RMG-open set in $\mathrm{X}$.

$\Rightarrow \mathrm{x} \in \mathrm{U}\{\mathrm{G}: \mathrm{G}$ is RMG-open, $\mathrm{G} \subset \mathrm{A}\}$.

$\Rightarrow \mathrm{x} \in \mathrm{RMG}-\mathrm{int}(\mathrm{A})$.

Thus, $x \in \operatorname{int}(\mathrm{A}) \Rightarrow \mathrm{x} \in \mathrm{RMG}$-int(A). Hence int(A) $\subset$ RMG-int(A).

Remark 5.9: Containment relation in the above Theorem 5.8 may be proper as seen from the following example.

Example 5.10: Let $X=\{a, b, c, d\}$ with topology $\tau=\{X, \emptyset,\{a\},\{b, c\},\{a, b, c\}\}$. Then $\operatorname{RMGO}(X)=$ $\{X, \emptyset,\{a\},\{b\},\{c\},\{a, b\},\{a, c\},\{b, c\},\{a, b, c\},\{a, b, d\},\{a, c, d\}\}$. Let $A=\{a, b\}$. Now RMG-int $(A)=\{a, b\}$ and $\operatorname{int}(A)=\{a\}$. It follows that int(A) $\subset$ RMG-int(A) and int(A) $\neq$ RMG-int(A).

Remark5.11: If $A$ is sub set of $X$, then

i) w-int(A)ᄃRMG-int(A).

ii) $\operatorname{g\alpha }-\operatorname{int}(\mathrm{A}) \subset \mathrm{RMG}-\mathrm{int}(\mathrm{A})$.

iii) $\mathrm{p}$-int(A)ᄃRMG-int(A).

Theorem5.12: If A is a subset of $X$, then RMG-int(A)ᄃMildly-g-int(A).

Proof: let A be a subset of a space $X$.

$\mathrm{x} \in \mathrm{RMG}-\mathrm{int}(\mathrm{A}) \Rightarrow \mathrm{x} \in \mathrm{U}\{\mathrm{G}: \mathrm{G}$ RMG-open, $\mathrm{G} \subset \mathrm{A}\}$.

$\Rightarrow$ There exists an RMG-open set $\mathrm{G}$ such that $\mathrm{x} \in \mathrm{G} \subset \mathrm{A}$

$\Rightarrow$ There exists an Mildly-g-open set $\mathrm{G}$ such that $\mathrm{x} \in \mathrm{G} \subset \mathrm{A}$, as every RMG-open set is an Mildlyg-open set in $X$.

$\Rightarrow \mathrm{x} \in \mathrm{U}\{\mathrm{G}: \mathrm{G}$ is Mildly-g-open, $\mathrm{G} \subset \mathrm{A}\}$.

$\Rightarrow \mathrm{x} \in$ Mildly-g-int(A).

Thus, $x \in$ RMG-int(A) $\Rightarrow x \in$ Mildly-g-int(A). Hence RMG-int(A) $\subset$ Mildly-g-int(A).

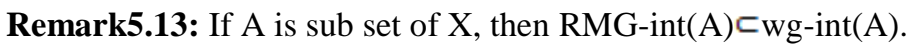

VI. Regular Mildly Generalized Closure (RMG-Closure) Operator.

Now we introduced the notation of RMG-closure in topological spaces by using the notation of RMGclosed sets and obtained some of their properties. For any A X, it is proved that the complement of RMGinterior of RMG-closure of the complement of A.

Definition 6.1: Let A be a subset of a space (X, $\tau$ ). We defined the RMG-closure of A to be a intersection of all RMG-closed sets containing A. In symbol we have RMG-cl(A)= $\{$ F:A $\subset F \in R M G C(X)\}$.

Theorem 6.2: Let $\mathrm{A}$ and $\mathrm{B}$ are subsets of $(\mathrm{X}, \tau)$. Then

i) $\mathrm{RMG}-\mathrm{cl}(\varnothing)=\varnothing$ and $\mathrm{RMG}-\mathrm{cl}(\mathrm{X})=\mathrm{X}$.

ii) $A \subset R M G-c l(A)$.

iii) If $B$ is any RMG-closed sets contained in $A$, then RMG-cl(A)ᄃB

iv) If $\mathrm{A} \subset \mathrm{B}$, then RMG-cl(A) $\subset \mathrm{RMG}-\mathrm{cl}(\mathrm{B})$.

v) $R M G-c l(R M G-c l(A))=R M G-c l(A)$.

Proof: i) Obvious.

ii) By the definition of RMG-closure of $\mathrm{A}$, it is obvious that $\mathrm{A} \subset \mathrm{RMG}-\mathrm{cl}(\mathrm{A})$. 
iii) Let B be any RMG-closed set containing A. Since RMG-cl(A) is the intersection of all RMG-closed set containing A, RMG-cl(A) is contained in every RMG-closed set containing A. Hence in particular RMG-cl(A)ᄃ B.

iv) ]Let $A$ and $B$ be subsets of $X$. such that $A \subset B$. By the definition of RMG-closure, RMG-cl(B) $=\cap\{F: B \subset F \in$ RMGC(X)\}. If $B \subset F \in R M G C(X)$, then RMG-cl(B)ᄃF. Since $A \subset B, A \subset B \subset F \in R M G C(X)$, We have RMG-cl(A)

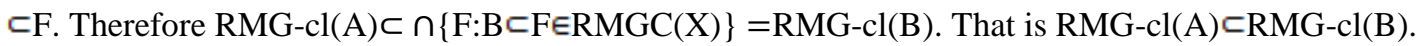

v) Since RMG-cl(A) is a RMG-closed set in $\mathrm{X}$, if it follows that RMG-cl(RMG-cl(A))=RMG-cl(A).

Theorem 6.3: If $A \subset X$ is RMG-closed, then $R M G-c l(A)=A$.

Proof: Let A be a RMG-closed subset of X. We know that AᄃRMG-cl(A). Also AᄃA and A is RMG-closed. By the theorem 6.2 (iii) RMG-cl(A)ᄃA. Hence RMG-cl(A)=A.

The converse of the above theorem need not be true as seen from the following example.

Example 6.5: Let $X=\{a, b, c, d\}$ with topology $\tau=\{X, \emptyset,\{a\},\{b\},\{a, b\},\{b, c\},\{a, b, c\}\}$. Then $\operatorname{RMGC}(X)=\{X, \varnothing,\{c\},\{d\},\{a, d\},\{c, d\},\{a, b, d\},\{a, c, d\},\{b, c, d\}\}$. Now RMG-cl $\{b\}=\{a, b, d\} \cap\{b, c, d\} \cap X=$ $\{b, d\}$, but $\{b, d\}$ is not a RMG-closed subset in $X$.

Theorem 6.7: If $A$ and $B$ are sub sets of $X$, then RMG-cl(A)U RMG-cl(B)ᄃRMG-cl(AUB).

Proof: Let $A$ and $B$ are subsets of a space $X$. Clearly $A \subset A \cup B$ and $B \subset A \cup B$. By Theorem6.2 (iv). RMG-

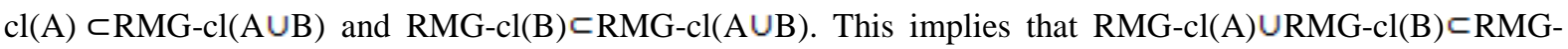
$\mathrm{cl}(\mathrm{A} \cup \mathrm{B})$.

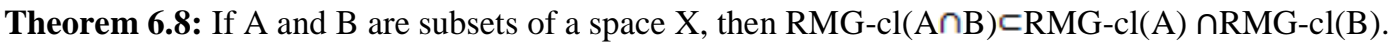

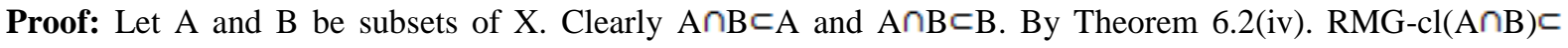

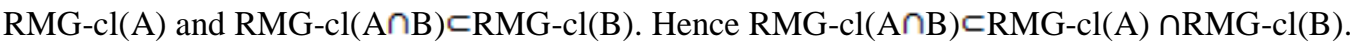

Theorem 6.9: Let $A$ be a subset of $X$ and $x \in X$. Then $x \in R M G-c l(A)$ if and only if $V \cap A \neq \emptyset$ for every RMGopen set $\mathrm{V}$ containing $\mathrm{X}$.

Proof: Let $x \in X$ and $x \in R M G-c l(A)$. To prove that $V \cap A \neq \emptyset$ for every RMG-open set $V$ containing $x$. Prove the results by contradiction. Suppose there exists a RMG-open set $V$ containing $x$ such that $V \cap A=\emptyset$. Then $A \subset X-$ $\mathrm{V}$ and $\mathrm{X}-\mathrm{V}$ is RMG-closed. We have RMG-cl(A)ᄃX-V. This shows that $\mathrm{x} \notin \mathrm{RMG}-\mathrm{cl}(\mathrm{A})$. Which is contradiction. Hence $\mathrm{V} \cap \mathrm{A} \neq \varnothing$ for every RMG-open set $\mathrm{V}$ containing $\mathrm{x}$.

Conversely, let $\mathrm{V} \cap \mathrm{A} \neq \varnothing$ for every RMG-open set $\mathrm{V}$ containing $\mathrm{x}$. To prove that $\mathrm{x} \in \mathrm{RMG}-\mathrm{cl}(\mathrm{A})$. We prove the result by contradiction. Suppose $\mathrm{x} \notin \mathrm{RMG}$-cl(A). Then there exists a RMG-closed subset $\mathrm{F}$ containing A such that $\mathrm{x} \notin \mathrm{F}$. Then $\mathrm{x} \in \mathrm{X}-\mathrm{F}$ and $\mathrm{X}-\mathrm{F}$ is RMG-open. Also $(\mathrm{X}-\mathrm{F}) \cap \mathrm{A}=\varnothing$, which is a contradiction. Hence $\mathrm{x} \in$ RMG-cl(A).

Theorem 6.10: Let $A$ be $A$ RMG-open set and $B$ be any open set in $X$. If $A \cap B=\emptyset$, then $A \cap R M G-c l(B)=\emptyset$.

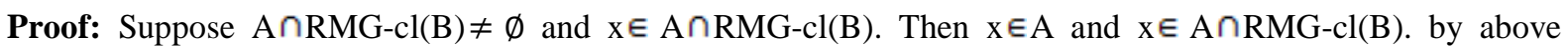
Theorem 6.9 $\mathrm{A} \cap \mathrm{B} \neq \varnothing$ which is contrary to the hypothesis. Hence AnRMG-cl(B) $\neq \emptyset$.

Theorem 6.11: If $\mathrm{A}$ is a subset of $(\mathrm{X}, \tau)$, Then $\mathrm{RMG}-\mathrm{cl}(\mathrm{A}) \subset \mathrm{cl}(\mathrm{A})$.

Proof: Let $A$ be a subset of $X$. By definition of closure, $c l(A)=\cap\{F \subset X: A \subset F \in C(X)\}$. If $A \subset F \in C(X)$, then $A \subset F$

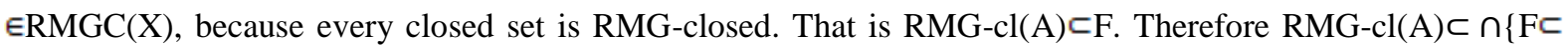
$\mathrm{X}: \mathrm{A} \subset \mathrm{F} \in \mathrm{C}(\mathrm{X})\}=\mathrm{cl}(\mathrm{A})$.Hence RMG-cl(A) $\subset \mathrm{cl}(\mathrm{A})$.

Remark 6.12: Containment relation in the above Theorem 5.34 may be proper as seen from the following example.

Example 6.13: Let $X=\{a, b, c, d\}$ with topology $\tau=\{X, \emptyset,\{a\},\{b\},\{a, b\},\{a, b, c\}\}$. Then $\operatorname{RMGC}(X)=\{X, \varnothing,\{c\},\{d\},\{c, d\},\{a, c, d\},\{b, c, d\}\}$. Let $A=\{c\}$. Now RMG-cl(A) $=\{c\}$ and $c l(A)=\{c, d\}$. It follows that RMG-cl(A)ᄃcl(A) and RMG-cl(A) $\approx \mathrm{cl}(\mathrm{A})$.

Remark6.14: If $A$ be a subset of space $X$, then

i) RMG-cl(A) $\subset_{W-c l}(\mathrm{~A})$.

ii) $\mathrm{RMG}-\mathrm{cl}(\mathrm{A}) \subset \mathrm{g} \alpha-\operatorname{cl}(\mathrm{A})$.

iii) $\mathrm{RMG}-\mathrm{cl}(\mathrm{A}) \subset \mathrm{p}-\mathrm{cl}(\mathrm{A})$.

Theorem 6.1:5 If A is a subset of space (X, $\tau$ ), Then Mildly-g-cl(A)ᄃRMG-cl(A).

Proof: Let A be a subset of X. By definition of Mildly-g -closure, Mildly-g-cl(A)= $\cap\{F \subset X: A \subset F \in$ Mildly-g-C $(\mathrm{X})\}$. If $\mathrm{A} \subset \mathrm{F} \in \mathrm{RMGC}(\mathrm{X})$, then $\mathrm{A} \subset \mathrm{F} \in \mathrm{Mildly}-\mathrm{g}-(\mathrm{X})$, because every RMG-closed set is Mildly-g-closed. That is

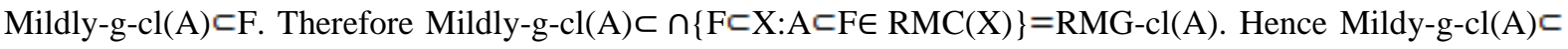
RMG-cl(A). 
Remark 6.16: If A subset of space X, then wg-cl(A)ᄃRMG-cl(A).

Lemma 6.17: Let $A$ be a subset of a space $X$. Then

i) $\quad \mathrm{X}-(\mathrm{RMG}-\operatorname{int}(\mathrm{A}))=\mathrm{RMG}-\mathrm{cl}(\mathrm{X}-\mathrm{A})$.

ii) $\quad \mathrm{RMG}-\operatorname{int}(\mathrm{A})=\mathrm{X}-(\mathrm{RMG}-\mathrm{cl}(\mathrm{X}-\mathrm{A}))$.

iii) $\mathrm{RMG}-\mathrm{cl}(\mathrm{A})=\mathrm{X}-(\mathrm{RMG}-\mathrm{int}(\mathrm{X}-\mathrm{A}))$.

Proof: Let $x \in X-(R M G-i n t(A))$. Then $x \notin R M G-i n t(A)$. That is every RMG-open set $U$ containing $x$ is such that $U \notin A$. That is every RMG-open set $U$ containing $x$ such that $U \cap(X-A) \neq \varnothing$. By the Theorem 6.9 , $x \notin R M G-$ $\mathrm{cl}(\mathrm{X}-\mathrm{A})$ and therefore $\mathrm{X}-(\mathrm{RMG}-\mathrm{int}(\mathrm{A})) \subset \mathrm{RMG}-\mathrm{cl}(\mathrm{X}-\mathrm{A})$.

Conversely, let $\mathrm{x} \in \mathrm{RMG}-\mathrm{cl}(\mathrm{X}-\mathrm{A})$, Then by Theorem 6.9, every RMG-open set $\mathrm{U}$ containing $\mathrm{x}$ such that $\mathrm{U} \not \subset \mathrm{A}$. This implies by definition of RMG-interior of $\mathrm{A}, \mathrm{x} \notin \mathrm{RMG}$-int $(\mathrm{A})$. That is $\mathrm{x} \in \mathrm{X}-(\mathrm{RMG}-\mathrm{int}(\mathrm{A}))$ and RMG-cl(X-A) and RMG-cl( $\left.\mathrm{A}^{\mathrm{C}}\right) \subset(\operatorname{RMG}-\operatorname{int}(\mathrm{A}))^{\mathrm{C}}$. Thus $(\mathrm{RMG}-\operatorname{int}(\mathrm{A}))^{\mathrm{C}}=\operatorname{RMG}-\mathrm{cl}\left(\mathrm{A}^{\mathrm{C}}\right)$.

ii) Follows by taking complements in (i).

iii) Follows by replacing $\mathrm{A}$ by $\mathrm{X}-\mathrm{A}$ in (i).

\section{References}

[1] D. Andrijevic, Semi-preopen sets, Mat. Vesnik, 38(1986), 24-32.

[2] S. S. Benchalli and R.S. Wali, On RW-closed sets in topological spaces, Bull.Malaysian.Math. Sci. Soc. (2) 30(2) (2007), 99-110.

[3] J. Dontchev and T. Noiri, Quasi-normal spaces and $\pi$-g-closed sets,Acta Math. Hungar.,89(3)(2000), $211-219$.

[4] T.Kong, R. Kopperman and P. Meyer, A topological approach to digital topology,Amer. Math. Monthly, 98 (1991), $901-917$.

[5] A.S. Mashhour, M.E. Abd. El-Monsef and S.N. El-Deeb, On pre continuous mappings and weak pre-continuous mappings, Proc Math, Phys. Soc. Egypt, 53(1982), 47-53.

[6] H. Maki, R. Devi and K. Balachandran, Associated topologies of generalized $\alpha$-closed sets and $\alpha$-generalized closed sets, Mem. Sci. Kochi Univ.Ser. A. Math., 15(1994), 51-63.

[7] H.Maki, J.Umehara and T.Noiri, Every topological space is pre-T1/2, Mem.Fac. Sci. Kochi. Univ. Ser. A. Math., 17(1966), 33-42.

[8] N.Levine, Semi-open sets and semi-continuity in topological spaces, Amer.Math. Monthly, 70(1963), 36-41

[9] N.Levine, Generalized closed sets in topology, Rend. Circ. Mat. Palermo,19(1970), 89-96.

[10] N. Nagaveni, Studies on Generalizations of Homeomorphisms in Topological Spaces, Ph.D.Thesis, Bharathiar University, Coimbatore, 1999

[11] O. Njastad, On some classes of nearly open sets, Pacific J. Math., 15(1965), 961-970.

[12] O. Ravi1*, I. Rajasekaran1 and M. Sathyabama2, Weakly g*-closed Sets, International Journal of Current Research in Science and Technology, Volume 1, Issue 5 (2015), 45-52.

[13] O. Ravi, S. Ganesan and S. Chandrasekar, On weakly $\pi$ g-closed sets in topological spaces, Italian Journal of Pure and Applied Mathematics (To Appear).

[14] N. Palaniappan and K.C.Rao, Regular generalised closed sets, kyungpook math, J.,33(1993), 211-219.

[15] A.Pushpalatha, Studies on generalizations of mappings in topolopgical spaces, Ph.D., Thesis, Bharathiar University, coimbatore(2000)

[16] J.K. Park and J.H. Park, Mildly generalized closed sets, almost normal and mildly normal spaces, Chaos, Solitions and Fractals, 20(2004), 1103- 1111

[17] M.Sheik John, On w-closed sets in topology, Acta Ciencia Indica, 4(2000),389-392.

[18] M. Stone, Application of the theory of Boolean rings to general topology,Trans. Amer. Math.Soc., 41(1937), $374-481$.

[19] N.V. Velicko, H-closed Topological Spaces, Tran. Amer. Math. Soc., 78 (1968), 103-118.

[20] R. S. Wali and Nirani Laxmi, On Regular Mildly Generalized (RMG) Closed set in topological spaces. International Journal of Mathematical Archive-Manuscript ref. No. 7212, 2016. 